\title{
Delayed surgical site infection after posterior cervical instrumented surgery in a patient with atopic dermatitis: a case report
}

\author{
Hiroshi Takahashi ${ }^{1}, 5$, Yasuchika Aoki ${ }^{2}$, Shinji Taniguchi ${ }^{1}$, Arata Nakajima ${ }^{1}$, Masato Sonobe ${ }^{1}$, \\ Yorikazu Akatsu ${ }^{1}$, Junya Saito ${ }^{1}$, Manabu Yamada ${ }^{1}$, Yasuhiro Shiga ${ }^{3}$, Kazuhide Inage ${ }^{3}$, \\ Sumihisa Orita ${ }^{3}$, Yawara Eguchi ${ }^{3}$, Satoshi Maki ${ }^{3}$, Takeo Furuya ${ }^{3}$, Tsutomu Akazawa ${ }^{4}$, \\ Masao Koda ${ }^{5}$, Masashi Yamazaki ${ }^{5}$, Seiji Ohtori ${ }^{3}$, and Koichi Nakagawa ${ }^{1}$ \\ ${ }^{I}$ Department of Orthopaedic Surgery, Toho University Sakura Medical Center, Japan \\ ${ }^{2}$ Department of Orthopaedic Surgery, Eastern Chiba Medical Center, Japan \\ ${ }^{3}$ Department of Orthopaedic Surgery, Chiba University Graduate School of Medicine, Japan \\ ${ }^{4}$ Department of Orthopaedic Surgery, St. Marianna University School of Medicine, Japan \\ ${ }^{5}$ Department of Orthopaedic Surgery, University of Tsukuba, Japan
}

\begin{abstract}
Objective: Atopic dermatitis (AD) is one of the known risk factors for Staphylococcus aureus infection. The authors report the case of a patient with cervical spondylosis and $\mathrm{AD}$ who developed delayed surgical site infection after posterior cervical instrumented surgery.

Patient: A 39-year-old male presented to our hospital with paralysis of the left upper extremity without any cause or prior injury. He had a history of severe AD. We performed C3-C7 posterior decompression and instrumented fusion based on the diagnosis of cervical spondylotic amyotrophy. One year after surgery, his deltoid and bicep muscle strength were fully recovered. Nevertheless, his neck pain worsened 2 years after surgery following worsening of AD. One month after that, he developed severe myelopathy and was admitted to our hospital. Radiographic findings showed that all the screws had loosened and the retropharyngeal space had expanded. Magnetic resonance imaging and computed tomography showed severe abscess formation and destruction of the C7/T1 vertebrae.

Result: We diagnosed him with delayed surgical site infection. Methicillin-resistant Staphylococcus aureus was identified on abscess culture. The patient responded adequately to treatment with antibiotic therapy and two debridements and the infection subsided.

Conclusion: We should consider the possibility of delayed surgical site infection when conducting instrumented spinal surgery in patients with severe AD.
\end{abstract}

Key words: surgical site infection, delayed infection, instrumented spinal surgery, atopic dermatitis

(J Rural Med 2020; 15(3): 124-129)

Received: March 5, 2020

Accepted: March 25, 2020

Correspondence: Hiroshi Takahashi, Department of Orthopaedic Surgery, Toho University Sakura Medical Center, 564-1 Shimoshizu, Sakura City, Chiba 285-8741, Japan

E-mail: hirochann@syd.odn.ne.jp

This is an open-access article distributed under the terms of the Creative Commons Attribution Non-Commercial No Derivatives (by-nc-nd) License <http://creativecommons.org/ licenses/by-nc-nd/4.0/>.

\section{Introduction}

Atopic dermatitis (AD) is a chronic inflammatory skin disease associated with cutaneous hyperactivity to environment triggers. Colonization by Staphylococcus aureus $(S$. aureus) is commonly observed in skin lesions of AD patients, and scratching the pruritic lesions leads to recurrent bacteremia $^{1)}$. Thus, AD is a known risk factor for $S$. aureus infection. Moreover, it is well known that AD is a risk factor for infectious endocarditis ${ }^{2,3)}$. In orthopedic literature, several reports have indicated that $\mathrm{AD}$ is a risk factor for septic arthritis of the hip, osteomyelitis, and septic bursitis of the olecranon $^{4-7)}$. Nevertheless, there are no reports of delayed 
surgical site infection after spinal surgery in patients with AD. Here, we report the case of a 39-year-old male with AD who had delayed surgical site infection following posterior cervical decompression and fusion.

\section{Case presentation}

A 39-year-old male reported to our hospital with paralysis of the left upper extremity following left scapula pain without any cause or injury. He had a medical history of severe AD. The cutaneous findings are exhibited in Figure 1. AD was treated with betamethasone ointment by a dermatologist at another hospital. At the initial visit, neurological examination revealed severe paralysis of the left deltoid (manual muscle test: MMT grade 1), biceps (MMT grade 2), and finger flexors and extensors (both MMT grade 4). The patient did not complain of sensory dysfunction. Radiographic findings showed dynamic instability at $\mathrm{C} 3 / 4$ and $\mathrm{C} 4 / 5$ (Figure 2A). Magnetic resonance imaging (MRI) showed spinal cord compression at $\mathrm{C} 3 / 4$ and $\mathrm{C} 6 / 7$ and left $\mathrm{C} 5$ root compression at $\mathrm{C} 4 / 5$. T2 hyperintensity was observed at the C3/4 level (Figure 2B). Furthermore, computed tomography (CT) myelography showed spinal cord compression at $\mathrm{C} 3 / 4$ and $\mathrm{C} 6 / 7$ due to ossification of the posterior longitudinal ligament (OPLL) (Figure 2C). We diagnosed the patient with cervical spondylotic amyotrophy and performed surgery. A C3-C7 posterior decompression and fusion with instrumentation (VERTEX, Medtronic Sofamor Danek, US) with C3-C6 laminoplasty and left C4/5 foraminotomy was performed (Figure 2D). Intraoperative findings showed severe left C5 nerve root compression. Decompression of the spinal cord and the left C5 nerve root was successfully performed. There were no severe complications, including surgical site infection. Laboratory investigations were normal 3 weeks after surgery, and the patient was dis- charged. His left upper extremity paralysis had significantly improved, and his deltoid and biceps strength were fully recovered one year after surgery. CT two years after surgery showed complete fusion from C3-7. Nevertheless, after two years of follow-up, the patient's AD had worsened. Subsequently, his neck pain also worsened, prompting a return to our hospital. There were no remarkable changes in his radiographic findings, and he was treated with collar fixation of the neck. One month after that, the patient developed severe myelopathy and gait disturbance and was re-admitted to our hospital.

On this admission, the patient's temperature was $38.0^{\circ} \mathrm{C}$. His neurological examination was notable for weakened finger extensors (MMT grade 2) and severe spasticity of both lower extremities. Radiographs showed that all screws were loosened and the retropharyngeal space had expanded (Figure 3A). MRI and CT showed severe abscess formation in the epidural space as well as the retropharyngeal space, plus destruction of the vertebrae at the C7/T1 level (Figure 3B, $3 \mathrm{C})$. Laboratory investigations were notable for $10.4 \times 10^{9} / \mathrm{L}$ white blood cell count, with $64 \%$ neutrophils, $13.17 \mathrm{mg} / \mathrm{dL}$ C-reactive protein (CRP), $76 \mathrm{~mm} /$ hour in the sedimentation test, and 1,580 IU/L creatinine kinase.

We diagnosed delayed surgical site infection based on neurological findings, imaging, and laboratory results. Implant removal, washout, and debridement was performed immediately. During the surgery, all implants were loosened and easily removed. A large amount of pus was noted to be emanating from the screw holes, and the diagnosis of infection was confirmed. After surgery, the patient was admitted to our hospital and was administered antibiotics. In addition, to control the $\mathrm{AD}$, the dermatologist in our hospital temporarily switched the steroid ointment from betamethasone to clobetasol. The treatment protocol with antibiotics is shown in Figure 4. Methicillin-resistant Staphylococcus
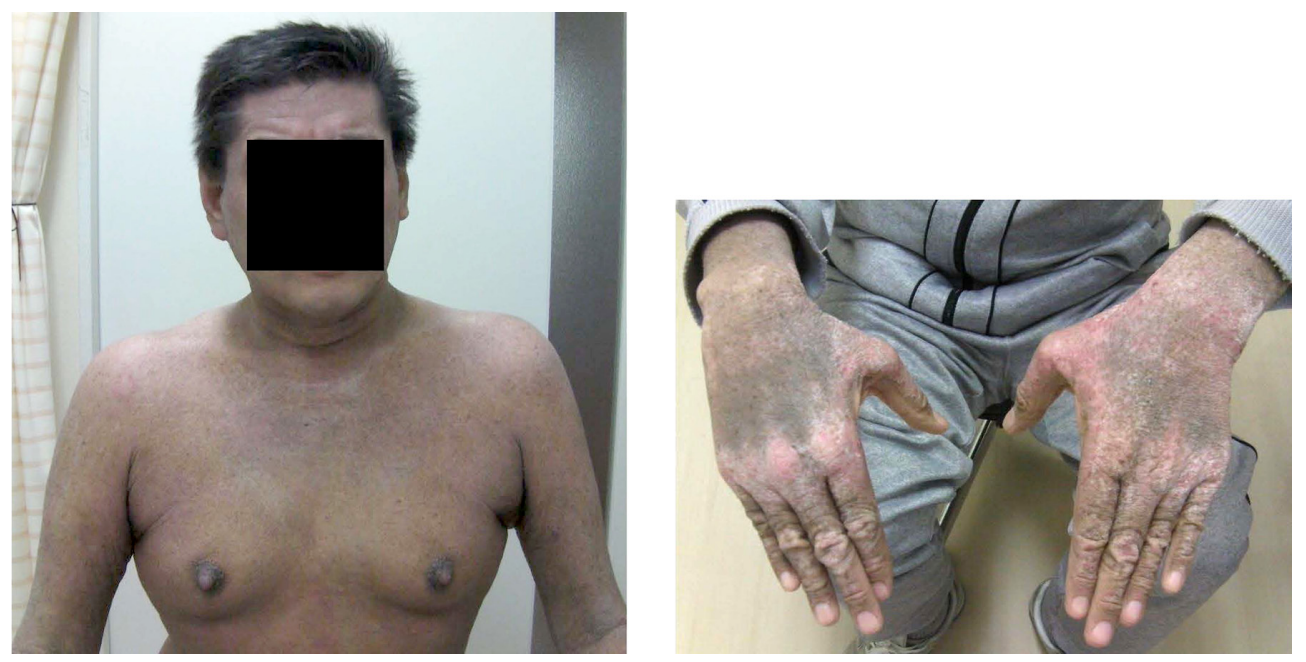

Figure 1 The patient's cutaneous findings. 
aureus (MRSA) was identified as a result of abscess culture, and treatment was based on the patient's susceptibilities. We added one more washout and debridement about 2 weeks after the initial implant removal surgery. Following this second surgery, the patient was noted to have responded adequately to treatment with antibiotics. Finally, both CRP and sedimentation test returned to the normal range, and the infection had subsided completely. We instructed bed
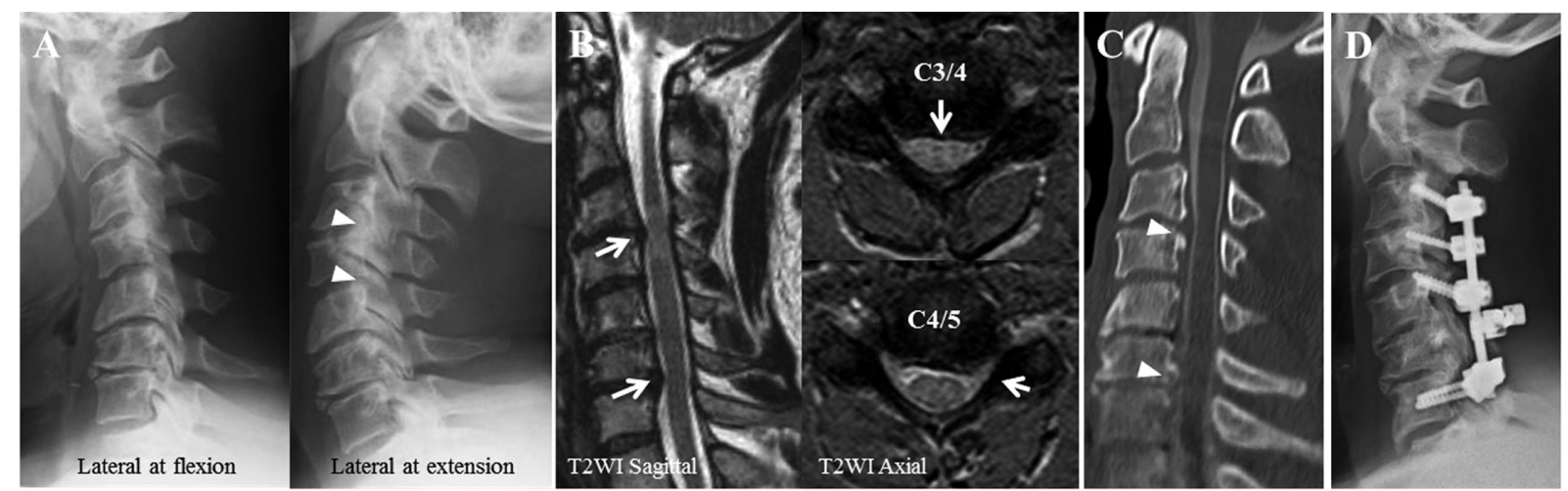

Figure 2 (A) Radiographic lateral functional image before surgery. Dynamic instability at C3/4 and C4/5 level was observed. (B) MRI T2WI. Sagittal imaging showed anterior indentation of the spinal cord at the C3/4 and C6/7 levels, and T2 hyperintensity was observed at the $\mathrm{C} 3 / 4$ level. Axial imaging showed spinal cord anterior indentation at the $\mathrm{C} 3 / 4$ and $\mathrm{C} 6 / 7$ levels and left nerve root compression at the $\mathrm{C} 4 / 5$ level (arrow). (C) CT myelography sagittal reconstruction imaging showed that the anterior indentation of the spinal cord was due to OPLL (arrowhead). (D) Radiographic lateral image after the primary surgery.
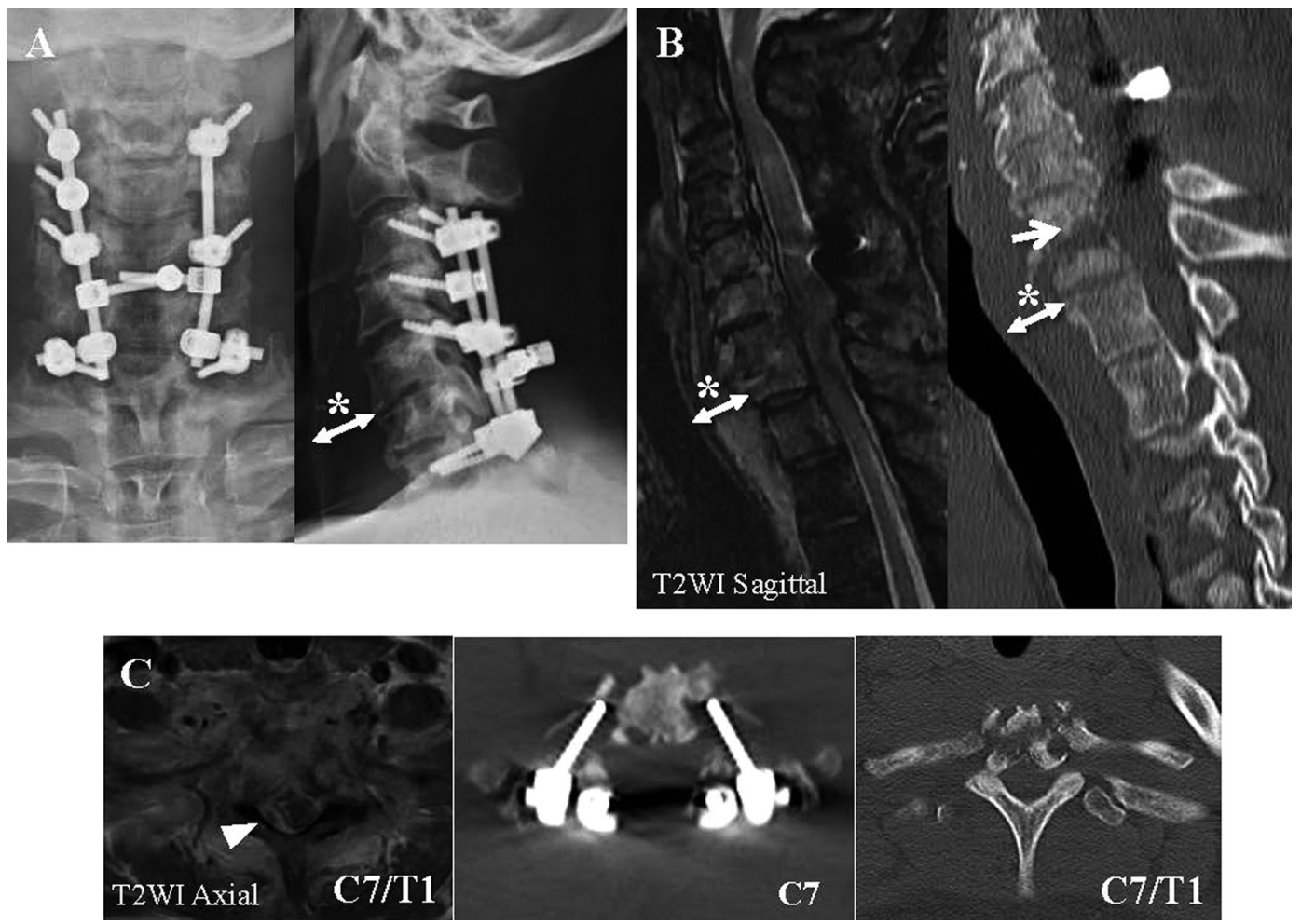

Figure 3 Imaging following worsened myelopathy. (A) Radiographic findings before implant removal. The retropharyngeal space was significantly expanded (asterisk), and radio-lucent areas were observed around the screws. (B) MRI T2WI and CT sagittal reconstruction. Asterisk shows severe abscess formation in the retropharyngeal space. CT sagittal findings show severe destruction of the C7-T1 vertebrae (arrow). (C) MRI T2WI axial view showing epidural abscess formation at the $\mathrm{C} 7 / \mathrm{T} 1$ level (arrowhead). CT axial view showing severe destruction of the $\mathrm{C} 7$ and $\mathrm{T} 1$ vertebrae around the loosened $\mathrm{C} 7$ pedicle screws. 


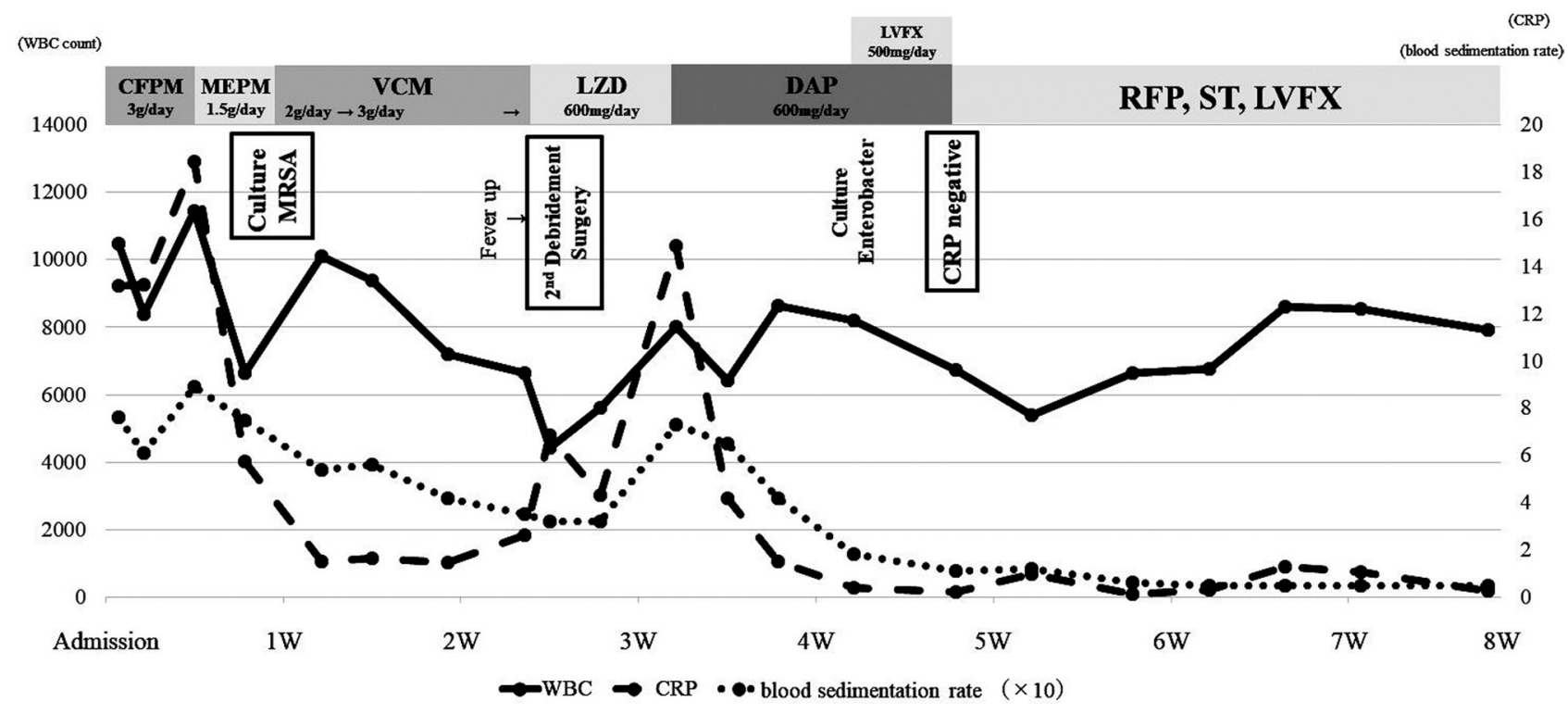

Figure 4 Laboratory changes and antibiotic treatment protocol after admission. CFPM: cefepime, MEPM: meropenem, VCM: vancomycin, LZD: linezolid, DAP: daptomycin, LVFX: levofloxacin, RFP: rifampicin, ST: sulfamethoxazole and trimethoprim.

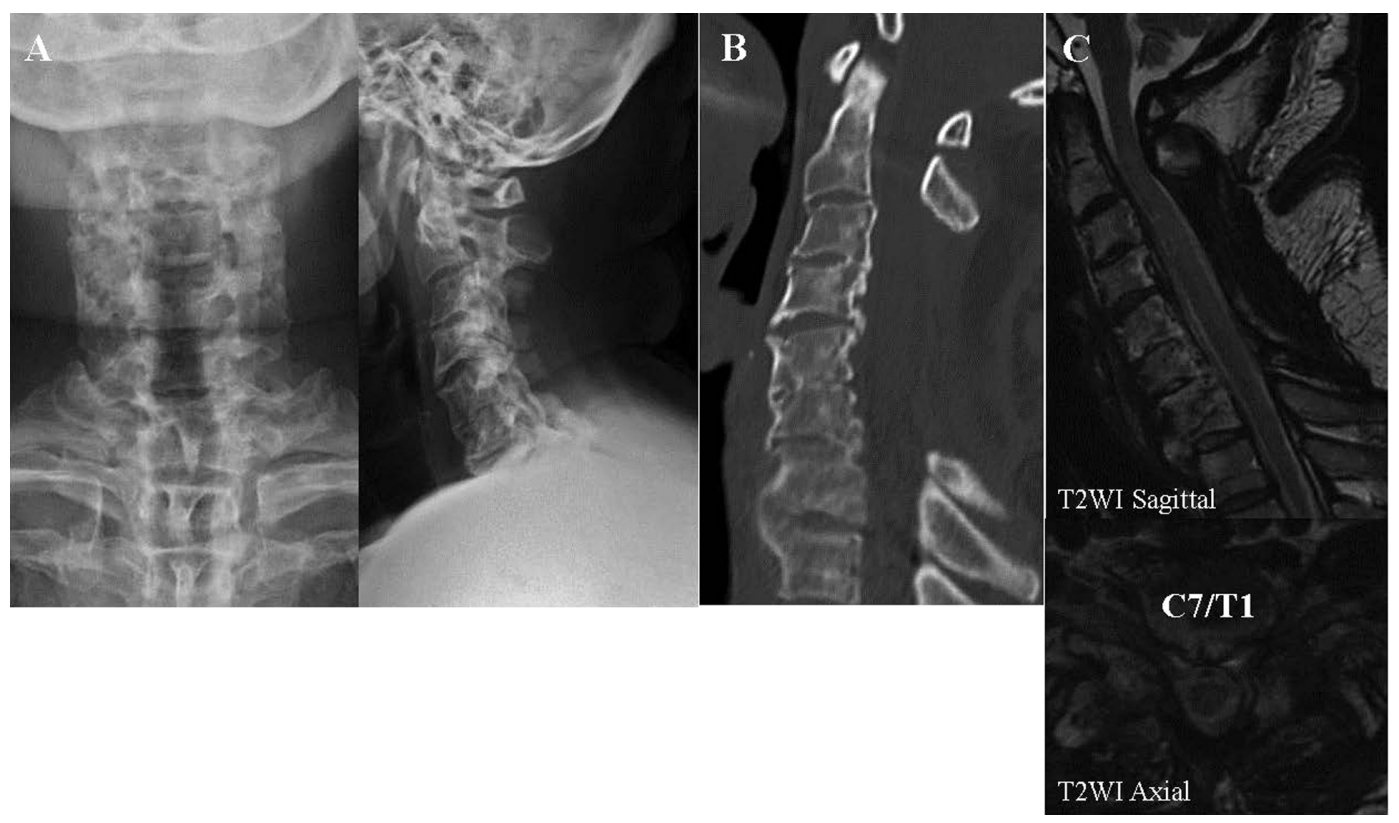

Figure 5 Imaging 2 years after surgery. (A) Radiograph showing retropharyngeal space contraction. (B) CT showing complete fusion of the C7/T1 vertebrae. (C) MRI T2WI showing improvement of epidural and retropharyngeal abscess and sufficient decompression of the spinal cord.

rest until the CRP returning to the normal range (that required 6 weeks) and 3 months collar immobilization to the patient. After rehabilitation, gait disturbance improved, and the patient was able to walk unassisted. Two years have passed since the surgery, and he has returned to his job. At the final follow-up, he was satisfied with passing the critical point; nevertheless, the final examination showed residual weakness of the third, fourth, and fifth fingers extension and abduction (MMT grade 3). Radiographs and CT two years after the last surgery showed complete fusion of the C7/T1 vertebrae (Figure 5A, 5B). MRI two years after surgery showed improvement of the epidural and retropharyngeal abscess and sufficient decompression of the spinal cord (Figure 5C). 


\section{Informed consent and ethical approval}

This case report study was conducted in accordance with the principles of the Declaration of Helsinki. Written informed consent was obtained from the patient for publication of this case report and any accompanying images. This study was approved by the ethical committee of Toho University Sakura Medical Center (S17007).

\section{Discussion}

To the best of our knowledge, this case is the first report of delayed surgical site infection after spinal surgery in an AD patient. According to the literature, the mechanism of $S$. aureus infection in AD entails first a decrease in lipid content of the stratum corneum of the epidermal layer of the skin, followed by incompetence of the skin barrier function, dry skin, and diaphoresis, and finally invasion of the body by the bacterium ${ }^{8}$. AD is therefore a known risk factor for $S$. aureus infection. It is reported that about $90 \%$ of AD patients are chronic carriers of $S$. aureus ${ }^{1}$. Several reports have indicated that $\mathrm{AD}$ is a risk factor for surgical site infection after heart surgery ${ }^{9)}$ and ophthalmic surgery ${ }^{10}$. These reports suggest that worsening of AD can be a cause of hematogenous seeding. Furthermore, there is one report of an orthopedic implant infection caused by $\mathrm{AD}^{11)}$. Similar to those reports, in our case, worsening AD resulted in hematogenous seeding, infection extended to around the screw holes, and severe abscess formation was eventually observed.

Surgical site infection after spinal fusion surgery is a relatively common complication ${ }^{12,13)}$. Once surgical site infection occurs following instrumented spinal surgery, it requires long-term antibiotic coverage, prolonged hospital stays, and multiple surgeries for debridement or drainage. This can result in a poor surgical outcome, and patients can develop adverse effects like chronic pain and paresthesias. Nevertheless, most infections occur early in the postoperative period, typically within the first 3 months. Infections occurring more than 3 months after surgery are rare ${ }^{14)}$ : the incidence of delayed surgical site infection following posterior instrumented spinal surgery is about $0.2 \%$, and the diagnosis can be challenging ${ }^{13}$. It has been reported that these delayed infections are associated with hematogenous seeding such as those occurring with intravenous drug use, urinary tract infection, neurogenic bladder, and pyelonephritis ${ }^{15,16)}$. In orthopedic lesions, hematogenous seeding is reported as a risk factor for delayed infection in total joint arthroplasty of the hip or knee ${ }^{17)}$. In our case, more than 2 years had passed following the first spinal fusion surgery, without early surgical site infection. There were no other underlying diseases that could have caused hematogenous seeding other than AD, and the symptoms worsened following exacerbation of AD. Furthermore, culture results confirmed MRSA, and thereby the occurrence of delayed surgical site infection. This is quite uncommon, and to the best of our knowledge, it represents the first case of delayed surgical site infection after instrumented spinal and fusion surgery due to $\mathrm{AD}$.

The use of prophylactic antibiotic therapy is reported to reduce the occurrence of delayed surgical site infection ${ }^{14}$. We may have to consider removing instrumentation to avoid delayed surgical site infection in such high-risk patients after complete bony fusion has occurred. Due to the infrequency of occurrence, there is no consensus on methods for preventing delayed surgical site infection. Further investigation using a large database is necessary.

In addition, to avoid severe delayed surgical site infection, surgical procedures without instrumentation may be considered for treating spine patients with severe AD. Several reports have indicated that using instrumentation is a risk factor for surgical site infection in spinal surgery ${ }^{18}$. Nevertheless, laminoplasty is not recommended for K-line (-) cervical OPLL: anterior decompression and fusion or posterior decompression and fusion (PDF) surgery, which both require instrumentation, are recommended ${ }^{19)}$. In this case, we chose PDF for the first surgical procedure because of severe dynamic instability at $\mathrm{C} 3 / 4$ and $4 / 5$ and concurrent OPLL. Although PDF was reasonable in this case, C3-7 laminoplasty with left $\mathrm{C} 4 / 5$ foraminotomy, which does not use instrumentation, might have been an option due to $\mathrm{K}$ line $(+)$ alignment in this patient before the primary surgery.

In conclusion, AD can be a cause of hematogenous seeding, and severe AD can be a major risk factor for early or delayed surgical site infection. We should consider the possibility of delayed surgical site infection, especially with MRSA, when planning instrumented spinal surgery in patients with severe AD.

\section{References}

1. Leung DY, Bieber T. Atopic dermatitis. Lancet 2003; 361: 151-160. [Medline] [CrossRef]

2. Mohiyiddeen G, Brett I, Jude E. Infective endocarditis caused by Staphylococcus aureus in a patient with atopic dermatitis: a case report. J Med Case Reports 2008; 2: 143. [Medline] [CrossRef]

3. Yamamoto T, Yodogawa K, Wakita S, et al. Recurrent prosthetic valve endocarditis caused by Staphylococcus aureus colonizing skin lesions in severe atopic dermatitis. Intern Med 2007; 46: 571-573. [Medline] [CrossRef] 
4. Benenson S, Zimhony O, Dahan D, et al. Atopic dermatitis - a risk factor for invasive Staphylococcus aureus infections: two cases and review. Am J Med 2005; 118: 1048-1051. [Medline] [CrossRef]

5. Boiko S, Kaufman RA, Lucky AW. Osteomyelitis of the distal phalanges in three children with severe atopic dermatitis. Arch Dermatol 1988; 124 : 418-423. [Medline] [CrossRef]

6. Kitamura S, Nakayama Y, Shirai Y, et al. Septic arthritis of the hip associated with atopic dermatitis. A case report. J Nippon Med Sch 2000; 67: 464-467. [Medline] [CrossRef]

7. Nassif A, Smith DL, Hanifin JM. Olecranon and pretibial bursitis in atopic dermatitis: coincidence or association? J Am Acad Dermatol 1994; 30: 737-742. [Medline] [CrossRef]

8. Cork MJ, Robinson DA, Vasilopoulos Y, et al. New perspectives on epidermal barrier dysfunction in atopic dermatitis: gene-environment interactions. J Allergy Clin Immunol 2006; 118: 3-21, quiz 22-23. [Medline] [CrossRef]

9. Fukunaga N, Yuzaki M, Shomura Y, et al. Clinical outcomes of open heart surgery in patients with atopic dermatitis. Asian Cardiovasc Thorac Ann 2012; 20: 137-140. [Medline] [CrossRef]

10. Oshima Y, Ohji M, Inoue Y, et al. Methicillin-resistant Staphylococcus aureus infections after scleral buckling procedures for retinal detachments associated with atopic dermatitis. Ophthalmology 1999; 106: 142-147. [Medline] [CrossRef]

11. Lim CT, Tan KJ, Kagda F, et al. Implant infection caused by dermatitis: a report of two cases. J Orthop Surg (Hong Kong) 2007; 15: 365-367. [Medline] [CrossRef]

12. Imajo Y, Taguchi T, Neo M, et al. Surgical and general complications in 2,961 Japanese patients with cervical spondylotic myelopathy: Comparison of different age groups. Spine Surg Relat Res 2017; 1: 7-13. [Medline] [CrossRef]

13. Collins I, Wilson-MacDonald J, Chami G, et al. The diagnosis and management of infection following instrumented spinal fusion. Eur Spine J 2008; 17: 445-450. [Medline] [CrossRef]

14. Lewkonia P, DiPaola C, Street J. Incidence and risk of delayed surgical site infection following instrumented lumbar spine fusion. J Clin Neurosci 2016; 23: 76-80. [Medline] [CrossRef]

15. Heggeness MH, Esses SI, Errico T, et al. Late infection of spinal instrumentation by hematogenous seeding. Spine 1993; 18: 492-496. [Medline] [CrossRef]

16. Viola RW, King HA, Adler SM, et al. Delayed infection after elective spinal instrumentation and fusion. A retrospective analysis of eight cases. Spine 1997; 22: 2444-2450, discussion 2450-2451. [Medline] [CrossRef]

17. Stinchfield FE, Bigliani LU, Neu HC, et al. Late hematogenous infection of total joint replacement. J Bone Joint Surg Am 1980; 62: 1345-1350. [Medline] [CrossRef]

18. Maruo K, Berven SH. Outcome and treatment of postoperative spine surgical site infections: predictors of treatment success and failure. J Orthop Sci 2014; 19: 398-404. [Medline] [CrossRef]

19. Koda M, Mochizuki M, Konishi H, et al. Comparison of clinical outcomes between laminoplasty, posterior decompression with instrumented fusion, and anterior decompression with fusion for K-line (-) cervical ossification of the posterior longitudinal ligament. Eur Spine J 2016; 25: 2294-2301. [Medline] [CrossRef] 